Multi Colors: An International Journal of

Educational Research and Theory Vol 1(1), 2018-19

\title{
Interprofessional Evidence-Based Practice Education: \\ Impact on Organizational Culture in Healthcare Settings
}

Sarah D. Eccleston ${ }^{1}$, Hannah Binder ${ }^{2}$, Lane Callahan ${ }^{3}$, Nancy S. Hodge $1^{1}$, and Mary S. McCarthy $1^{1}$, Madigan Army Medical Center, Tacoma, WA

\footnotetext{
${ }^{1}$ Center for Nursing Science \& Clinical Inquiry

${ }^{2}$ Critical Care Nursing Section

${ }^{3}$ Medical-Surgical Nursing Section
}

Disclaimer: The views expressed in this paper are those of the authors and do not reflect the official policy of the Department of the Army, the Department of Defense, or the U.S. Government. Correspondence concerning this article should be addressed to Mary S. McCarthy, 1611 Nisqually St., Steilacoom, WA 98388. Email: mary.s.mccarthy1.civ@mail.mil

\begin{abstract}
A key strategy for improving the quality of health care is through the widespread implementation of evidence-based practice (EBP). Most importantly, EBP helps organizations achieve high reliability and a culture of safety. Research shows that EBP reduces morbidity, mortality, medical errors, and geographic variation in health care. This paper describes an interprofessional education intervention to transform clinical care and institutionalize EBP in one medical facility. Through the adoption of an intensive EBP training platform and the rapid implementation of EBP solutions to address traditional challenges, the organization is effectively accelerating its efforts to move from a health care system to a highly reliable system of health..
\end{abstract}

Keywords evidencebased practice, high-reliability organization, quality improvement, interprofessional education 


\section{Introduction}

Health professionals who are educated to understand each other's roles and the value of teamwork are better equipped to work collaboratively and to deliver high-quality care with improved patient outcomes (Cartwright, Franklin, Forman, \& Freegard, 2015). The development of an effective team was identified years ago by the Institute of Medicine (IOM, 2001) as one of the six steps needed to reinvent the healthcare system and improve patient care. Additionally, the IOM identified 10 strategies necessary to support this process, including care customized to the patient's needs and values with evidence-based decision making. Research shows that implementing evidence-based practice (EBP) improves the quality and safety of health care, enhances health outcomes, decreases geographic variation in care, and reduces costs (McGinty \& Anderson, 2008; Melnyk \& Fineout-Overholt, 2015; Melnyk, Fineout-Overholt, Gallagher-Ford, \& Kaplan, 2012; Shortell, Rundall, \& Hsu, 2007; Williams, 2004). However, research also shows that only one in four health professionals provides care within an EBP framework (Patelarou et al., 2017). One strategy key to improving the quality of care is through the widespread implementation of EBP, which helps organizations attain high reliability. This problem-solving approach to care delivery integrates the best evidence from well-designed studies with a clinician's expertise, including clinical wisdom, reasoning, patient history, physical data collection, and resource use, and the patient's preferences and values to make decisions about the type of care to provide (Melnyk \& Fineout-Overholt, 2015). Key factors facilitating EBP adoption include strong beliefs that EBP improves patient care and outcomes, a solid foundation of knowledge and skills, access to EBP mentors, and an organizational culture that supports evidencebased care (Melnyk \& Fineout-Overholt, 2015). Furthermore, studies indicate that implementing EBP leads to improved patient outcomes by at least $28 \%$; the U.S. healthcare system could reduce healthcare spending by $30 \%$ if patients consistently receive evidence-based care (Buntin et al., 2006; Melnyk, 2012). Melnyk and FineoutOverholt (2002) developed the Advancing Research and Clinical Practice with Close Collaboration (ARCC) model (Fig. 1). The model provides a framework for implementing professional learning through EBP activities throughout the organization. This systematic process has been shown to sustain EBP, improve outcomes, and achieve high reliability (Melnyk, 2012; Melnyk \& Fineout-Overholt, 2015). The interprofessional education platform described in this paper is an example of an organization-wide strategy to introduce a comprehensive curriculum based on the ARCC model that promotes a culture of inquiry to foster high-quality, evidence-based, patient-centered care. 


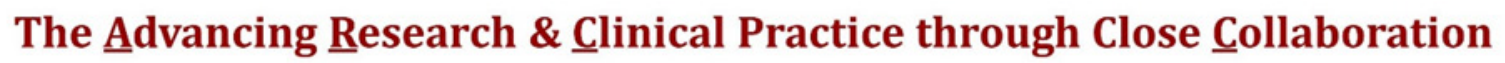
(ARCC) Model

CC Melnyk \& Fineout-Overholt, 2005

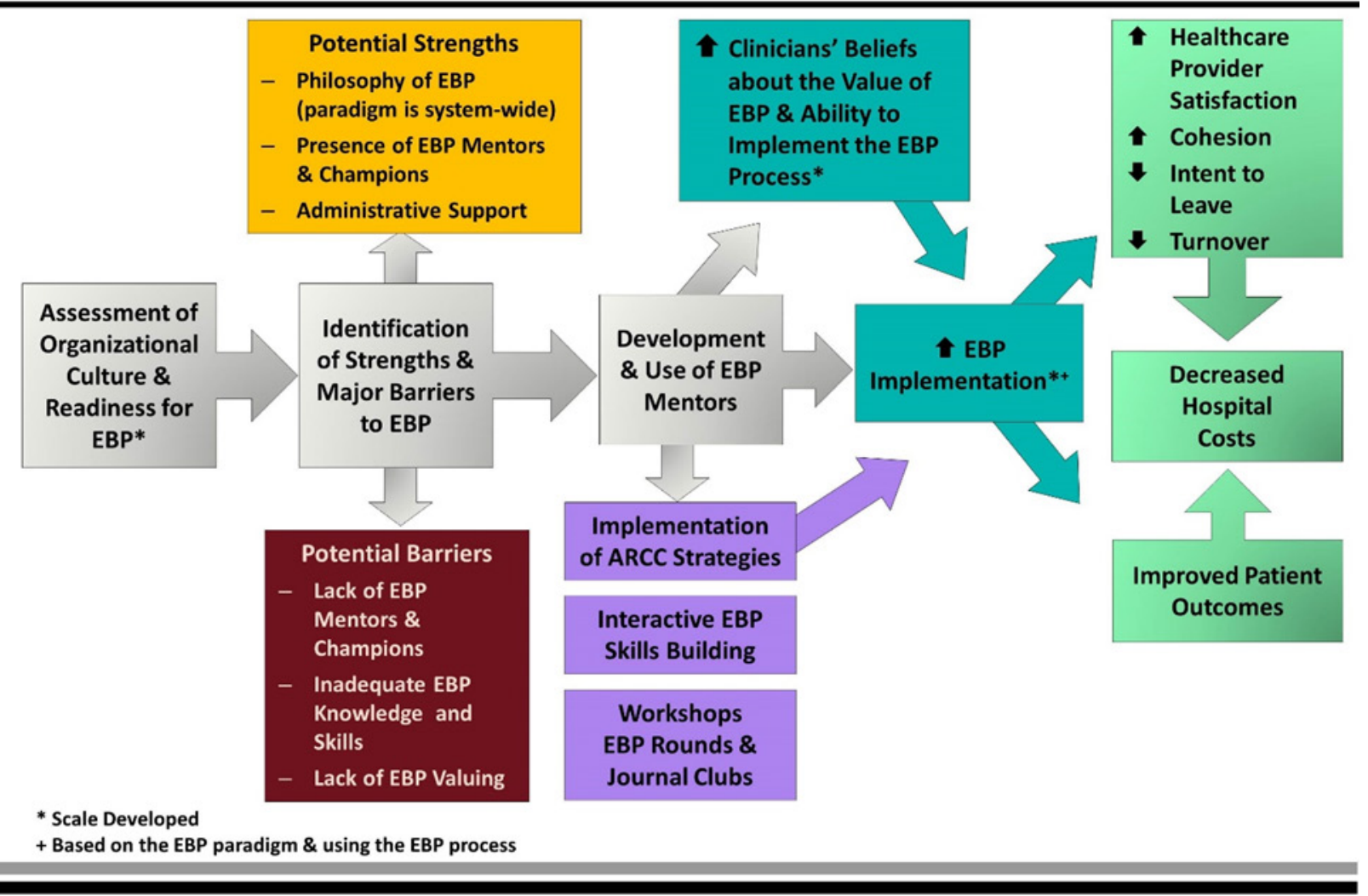

Fig. 1. ARCC model to guide organizational evidence-based practice implementation. Reproduced from B. M. Melnyk and E. Fineout-Overholt, Evidence-Based Practice in Nursing \& Healthcare (2nd ed.), p. 291, Philadelphia, PA: Wolters Kluwer / Lippincott Williams and Wilkins Health, copyright Wolters Kluwer Health, 2015. Reproduced with permission

\section{Organizational Culture}

2.1. High-reliability organizations

In the decade following the report from the Evidence-Based Medicine Roundtable (IOM, 2009),

hospital organizations have continued to face heavy scrutiny for lapses in safety and quality, with accrediting bodies demanding more stringent infection control and medication safety standards. Reports in the lay press suggested that medical errors continued to cause over 400,000 deaths each year (James, 2013; McCann, 2014). The IOM report (2009) stated, in part:

We seek the development of a learning healthcare system that is designed to generate and apply the best evidence for the collaborative healthcare choices of each patient and provider; to drive the process of discovery as a natural outgrowth of patient care; and to ensure innovation, quality, safety, and value in health care. (p. 1)

About the same time as the IOM report (2009), the highest office governing U.S. Army Medicine began the transformation from a healthcare system to a system for health; the new focus was on prevention of disease, injury, and disability going forward. This call to action involved developing the organization's infrastructure to 
encourage healthcare consumers, such as active and retired service members and their families, to develop a mindset of self-care (Horoho, 2013). The Army community at large was introduced to a health promotion model with three pillars-activity, nutrition, and sleep-as the foundation for optimal health and resilience in the face of military-unique challenges. To meet the system for health imperatives, the top executives of Army Medicine began the journey toward becoming a high-reliability organization (HRO) because its safety and quality focus, along with favorable health outcomes and customer satisfaction, resonated across the Army medical enterprise. "High-reliability science is the study of organizations in industries like commercial aviation and nuclear power that operate under hazardous conditions while maintaining safety levels that are far better than those of health care" (Chassin \& Loeb, 2013, p. 459). The Army-specific HRO theory asserts that its framework will provide opportunities to apply best practice ideas in a healthcare setting, allow staff to develop deeper understanding of what Army Medicine must do to provide the best possible care to patients, to improve retiree and family care, and to create a safe environment for patients and staff (D. McKay, personal communication, August 22, 2017). The path to becoming an HRO is not a familiar one for hospitals, and military hospitals are no exception. Hospitals have a poor reputation for reliability and grave deficiencies in safety, yet little was done to change this public opinion until the Joint Commission (2008) required hospital leaders of healthcare organizations to adopt and maintain a culture of safety. The Joint Commission provides accreditation for hospitals meeting the highest standards of care and endorses the HRO framework, but it cautions that a series of incremental steps should be followed by any organization seeking high-reliability healthcare operations. These steps include leadership commitment to achieving zero patient harm, a fully functional culture of safety throughout the organization, and widespread deployment of highly effective process improvement (PI) tools (Chassin \& Loeb, 2013). These tools are typically embedded in the organization and reflect the health system, its mission, its priorities, and the patient population it serves. The use of EBP frameworks to address PI or quality improvement (QI) deficiencies is no different for military hospitals, yet the highly mobile workforce, the generally younger health care clients, and the mission to support warfighter readiness present some unique challenges. At Madigan Army Medical Center, we set out on a path to becoming an HRO whereby top leaders demonstrate a commitment to organizational change, a safety culture, and robust PI methodologies (Weick \& Sutcliffe, 2007). The combined efforts not only empowered healthcare beneficiaries but also engaged healthcare professionals in adopting evidence-based practices and safety and quality initiatives across all care delivery settings.

\subsection{A case study using a hospital with a military infrastructure}

There are five common principles of an HRO all of which must be addressed for success in any organization, and they can be applied to providing safe, effective care with a goal of eliminating all serious patient harm in hospitals (Weick \& Sutcliffe, 2007). These are preoccupation with failure, reluctance to simplify, sensitivity to operations, commitment to resilience, and deference to expertise. In addition, the founders of this approach state that it is important for the organization to exhibit a "collective mindfulness," which means encouraging the identification of errors or close calls for the lessons learned through careful analysis and strategies identified to strengthen protocols and reduce failure (Weick \& Sutcliffe, 2007). This case-study organization established an office devoted to developing and implementing interactive interprofessional training to provide clarity and real-world examples for each of the five guiding principles. Madigan Army Medical Center is the second-largest medical center in the U.S. Army and one of four level II-accredited trauma centers in Washington State, with about 220 inpatient beds and a patient-care mission executed by over 5,000 staff members, including hundreds of civilian and military doctors, nurses, pharmacists, dietitians, physical therapists, and social workers. In addition, it offers are over 30 graduate medical and nursing education programs involving 530 trainees. Each day there are 30 new admissions, eight newborn deliveries, and 45 surgeries performed; the potential for patient harm is ever present (Madigan Army Medical Center, 2018). The diversity in patient populations and the multitude of clinicians, all possessing different licenses and skills, speaks to the need for common interprofessional education 
efforts in support of teamwork and patient-centered care. An organization is not considered an HRO unless it can demonstrate continuing success in striving for zero preventable harm in an environment where human error is possible and accidents can and do occur related to risk factors and complexity.

\subsection{Interprofessional evidence-based practice education}

Interprofessional education is a framework that applies to many different work or academic environments. It has become apparent that its application in health care is not only appropriate but necessary. There is a new shift in the clinical environment, with support for interprofessional collaborative practice, where healthcare providers from multiple professions synchronize their care to deliver patient-centered, high-quality care (World Health Organization [WHO], 2010). These collaborations have been shown to improve patients' access to care and health outcomes and to decrease patient complications and clinical errors (WHO, 2010). As a result, there is an increased need for young, inexperienced clinicians representing different professions to be trained together in the clinical setting in order to better understand how to best work together and to meet the core competencies for interprofessional collaborative practice (Interprofessional Education Collaborative, 2011). While new nurses, doctors, or other health professionals may learn the concepts around interprofessional collaboration in the classroom or in simulations, having a place to apply the skills with guidance and role modeling from an experienced clinician/preceptor is essential for their skill and role development. The following literature review provides exemplars of interprofessional collaboration in the healthcare setting.

\section{Literature Review}

Interprofessional education (IPE) is a pedagogical approach commonly used for preparing health professions students to provide patient care in a collaborative team environment. Several authors have stated that an appealing premise of IPE is that, once healthcare professionals work in a collaborative manner, patient care improves (Barr, Koppel, Reeves, Hammick, \& Freeth, 2005; Freeth, Hammick, Reeves, Koppel, \& Barr, 2005; Brashers, Curry, Harper, McDaniel, Pawlson, \& Ball, 2001). Interprofessional teams reportedly enhance the quality of patient care, lower costs, decrease patients' length of stay, and reduce medical errors (AHRQ, 2008). Blewett, Johnson, McCarthy, Lackner, and Brandt (2010) described inpatient geriatric services delivered by interprofessional teams as resulting in an average decrease in hospital costs of $\$ 2,000$ per patient and a decrease in length of stay by 7 days compared with units without interprofessional teams.

The IOM (2003), WHO (1988), and the National Academies of Practice (Brashers et al., 2001), among others, endorsed IPE as a strategy that improves the overall quality of health care. Interprofessional education models are easily adapted to different learning and care delivery environments; the implementation can be effective whether in the classroom, simulation laboratory, or hospital unit. Cartwright et al. (2015) used an online case study about dementia with 125 health science students to encourage collaborative interprofessional capabilities and a client-centered mindset. They found that mean scores on the Interprofessional Socialization and Valuing Scale (King, Shaw, Orchard, \& Miller, 2010), which measures a shift in behaviors and attitudes, improved significantly (preintervention $=107.79, \mathrm{SD}=14.24$; postintervention $=115.62, \mathrm{SD}=13.80 ; \mathrm{p}<.001$ ) with case-study completion. Students described a better understanding of other health professions and the importance of team collaboration. The authors commented that health professionals who are trained to understand each other's roles and the value of teamwork are better equipped to work collaboratively and to deliver high-quality care with improved patient outcomes. In another study, Chen et al. (2017) used a combination of IPE teaching methods, including online modules, and face-to-face small group sessions to develop skill and knowledge competencies for over 500 health professions students. The students reported that while the value of the online modules was "good," the opportunity to interact with their interprofessional peers was more meaningful and valued more highly. 
This blended IPE model effectively addressed many barriers plaguing IPE curricula such as class scheduling, space and time for training, matching levels of learners, faculty development, and institutional buy-in (Chen et al., 2017).

Faculty in one healthcare setting implemented an IPE approach for health professions students to design longitudinal care management for patients with uncontrolled diabetes (Coleman, McLean, Williams, \& Hasan, 2017). Faculty members were based in both an ambulatory clinic in an urban hospital and an urban family medicine practice. The investigator evaluated the attitudes toward teamwork and patient perceptions of care delivery. Teamwork skills significantly improved for students participating in the program, and attitudes toward teamwork also improved, but not significantly. On patient surveys, the patients $(\mathrm{n}=24)$ who were actively engaged in the program reported higher satisfaction with care delivery than control patients did $(\mathrm{n}=$ 228). In a prospective, descriptive, mixed-methods study (Fisher, Weyant, Sterrett, Ambrose, \& Apfel, 2017) involving clinicians in a large pediatric hospital, a survey was used to determine perceptions of interprofessional collaboration, and the researchers evaluated the relationship of select items on the Collaborative Practice Assessment Tool (CPAT) with the Press Ganey (PG) patient satisfaction survey. Results indicated a moderately high perception of interprofessional collaborative team practices $(\mathrm{M}=5.51, \mathrm{SD}=0.75)$ among direct patientcare providers $(n=173$, or a $13 \%$ response rate) throughout the hospital. The highest perceptions were noted in the domains of Patient Involvement $(\mathbf{M}=6.18, \mathrm{SD}=0.95)$ and Decision Making $(\mathrm{M}=4.53, \mathrm{SD}=0.82)$. There was a trend toward significance between professions of medicine and nursing regarding perceptions of collaborative teamwork; no ancillary staff participated. Demographics such as age, education, and experience did not significantly affect CPAT scores; however, the cohort with the most years of experience had the highest scores. There was no relationship between average CPAT score and responses on the PG patient satisfaction survey $(\mathrm{r}=0.009, \mathrm{p}=0.964)$. The qualitative responses expressed a common theme, with all clinicians having a desire for a respectful, positive work environment.

A unique approach to the implementation of IPE is that of Peterson and Brommelsiek (2017), who described an IPE course offered as an 8-week immersion experience. Faculty facilitated discussion and provided scenario-based exemplars of EBP interventions, while students grappled with progressively more challenging scenarios requiring a higher degree of interprofessional collaboration. Students were also scheduled for clinical practicums in the Veteran's Administration hospital in teams of two to three, along with one faculty member. Goals of the clinical practicum were to promote team building with patients and other clinicians, develop confidence in using interprofessional resources, build resilience, and help students commit to a patient-centered plan of care. Students maintained journals, which were used along with interviews to evaluate the course. In the end, students increased their knowledge of, confidence in, and attitudes about working with interprofessional teams; they also increased their comfort and situational control, their self-efficacy, and their commitment to patient-centered care. One opinion expressed by the authors was that health professionals will develop a stronger sense of social resilience if they are able to build mutual trust and communication skills through exposure to IPE and interaction with healthcare teams throughout their curriculum.

A recent report in the literature describes a typical ICU environment with dynamic interpersonal communication between physicians and nurses during afternoon rounds and hand-offs. Using 16 semistructured interviews and nine field observations, Serksnys, Nanchal, and Fletcher (2017) reported that physicians were interested in information about family dynamics, changes in patient condition, and patient data in the context of the clinical situation. Nurses expressed interest in information about technical data, anticipatory guidance, and the goals for the plan of care. Both the physicians and nurses in this medical ICU placed a high value on interprofessional communication, and both groups felt that it was important to incorporate into the hand-off communication.

Last, a published report of a QI approach (Makic \& Wald, 2017) to a clinical issue involving an interprofessional team is similar to the process underway here at Madigan Army Medical Center. The authors describe the steps taken to define a process for improvement; establish an interprofessional team based on expertise and clinical role, such as providers, nurses, quality experts, and informaticists, and other stakeholders, such as the infection preventionist and a logistician; and develop a plan. The team must choose a leader, decide on an appropriate meeting schedule, and review the basics that distinguish QI from research or implementation 
science so that all team members have a clear understanding of the plan. The team must then select relevant outcome measures and determine who will collect data and manage the database, how it will be documented, and where will it be maintained for privacy and security reasons. The remaining steps involve guidelines for clear and effective communication, cleaning and analyzing data, and, finally, prioritizing dissemination activities. Below, we discuss Madigan Army Medical Center's preference from numerous available models to guide the transformation to a culture that endorses IPE and encourages EBP within a HRO.

\section{One Organization's Adoption of an Evidence-Based Practice Model}

On the journey to becoming a HRO, our hospital strongly endorsed the recommendation by experts to adopt robust PI tools and methodologies. Advanced practice nurses on the hospital staff introduced an innovative intervention that held the potential to transform organizational culture in alignment with HRO principles. The overall goal of the program, developed by the Center for Transdisciplinary Evidence-Based Practice (CTEP), is to help healthcare organizations rapidly translate research and clinical data into practice with an evidence-based approach. This approach enables staff to provide supportive, evidenced-based care to drive higher quality, safety, and consistency of health care, and to reduce costs. The CTEP approach uses strategies, specifically designed to enhance systemwide implementation and sustainability of evidence-based care, that are multipronged and engrained throughout the organization. Adopting the CTEP approach to develop EBP mentors was in line with the IOM's 2020 goal of ensuring that $90 \%$ of clinical decisions are based on evidence (IOM, 2009). Experts leading the IOM Roundtable on Evidence-Based Medicine discussion expressed that this goal presented a tangible focus for progress toward the vision of a healthy America, that Americans ought to expect at least this level of performance, that it should be feasible with existing resources and emerging tools, and that outcome measures should be developed to track and stimulate progress (IOM, 2009).

The CTEP program was developed by leaders in the field of evidence-based nursing practice who have published extensively on the implementation of EBP (Gallagher-Ford, 2014; Melnyk \& Fineout-Overholt, 2011; Melnyk, Fineout-Overholt, Gallagher-Ford, \& Kaplan, 2012; Titler et al., 2001). The faculty and support staff for CTEP engage learners from professional disciplines within the participating institution. The program is customizable and dynamic, which allows the necessary flexibility for a military healthcare operation where uncertainty due to short-notice requirements, deployments, or budget cuts often dominates the day-to-day tempo. The 5-day comprehensive curriculum takes into consideration the busy interprofessional audience. Data from prior IPE EBP programs suggest that providing different options for teaching EBP results in increased engagement of professionals with expected individual learning styles and preferences (Patelarou et al., 2017). Recent efforts at our institution to pair nurses and physicians working in the same patient-care setting where an identified knowledge gap or patient-care issue required attention supported the core elements of interprofessional collaborative education. Arriving at the course prepared with a clinical question or a near-miss/actual safety event was also key to accomplishing the lofty goal of designing an EBP project in 5 days.

Participants in the program learn how to formulate a clinical question, search and find evidence in the literature, critically appraise the literature, summarize the evidence, and plan and implement an EBP change within the organization. Other community courses on EBP typically review the information on the conceptual level without including skills for application. The course instructors and facilitators assist the organization by offering one-on-one mentorship to enable attendees to develop a project that is a high priority for the organization as well as for the clinician and their work setting. Based on the education provided, participants receive 36.5 continuing education credits upon completion, which is important for licensure and performance appraisals in our organization. Adoption of specific EBP competencies for nurses, advanced practice nurses, physicians, dietitians, and other healthcare professionals who practice in real-world settings can support institutions in 
achieving high-value, low-cost, evidence-based health care. Regardless of the organization, the culture in which nurses practice affects the success of engagement in and sustainability of EBP. Therefore, it is imperative for the organization's leaders to invest in creating a culture of inquiry and an environment to support clinical excellence through EBP. Furthermore, by investing in this culture and this approach to informed practice, leaders are also encouraging communication and teamwork, which are critical to eliminating harm, promoting safety and quality, and achieving high reliability in the hospital setting. Through the endorsement of this 5-day intensive EBP education platform and the implementation of high-impact EBP changes, this organization is effectively accelerating its efforts to move from a healthcare system to a highly reliable system of health. According to Chassin and Loeb (2013), in HROs, quality and safety are the personal responsibility of every employee, and being taught how to use EBP to solve complex problems gives employees confidence to exercise that responsibility.

\subsection{Implementing an interprofessional educational intervention}

Through a contractual arrangement approved by top leaders in the case-study hospital, CTEP

faculty provided 5-day on-site IPE on four occasions. To avoid scheduling conflicts and competing priorities with organizational initiatives, the program was planned 6 months in advance with a spring and fall course. A total of 112 professionals have attended the course, with work initiated on numerous projects; ongoing efforts actively support 22 projects as of this writing. Practice innovations that are a direct result of the IPE EBP training efforts are highlighted below.

- Lack of effective communication between care team members, namely physicians and nurses, regarding the patient's plan of care resulted in more frequent pager calls, confusion among team members when addressing patient and ancillary staff inquiries, and a reduction in patient satisfaction scores on standard post-hospitalization surveys. The implementation of multidisciplinary rounds attended jointly by nurses and physicians on one inpatient medical-surgical unit reduced pager calls by $20 \%$ over 3 months, improved the staff's understanding of the plan of care, and increased patient satisfaction scores.

- Building on the idea that understanding other healthcare team members' roles and responsibilities could positively influence communication, respect, and patient care, one nursing unit developed a job-shadow experience where each nurses spent a day following a physician and, on a separate day, the physician followed that nurse through his/her shift. This activity enhanced teamwork and gave professionals more appreciation for each other's busy schedule and patient-care responsibilities.

These are just two examples of how incorporating the transdisciplinary EBP model into practice addressed important role identity and patient-care issues and relied on teamwork and HRO principles to achieve overwhelming success. These projects will require continuous support from the executive leadership and unit champions, as well as the physician and nursing staff, to overcome the barriers to sustaining practice change in a complex and unpredictable healthcare environment.

\subsection{Sustainment challenges and solutions}

As mentioned, military-unique considerations such as a highly mobile workforce and short-notice mandates for deployment, curtailment of operations, or shifting priorities have a direct and measurable impact on newly adopted practice changes. Once the current leaders transfer to new locations and positions, the incoming leaders may have a personal vision for innovation and change that redirects resources, personnel, or services rendered, resulting in new or revised strategies to accomplish the mission. In a military hospital it is not uncommon for senior leaders to relocate every 2 to 3 years, which affects the professional staff at all levels. Middle managers who support EBP approaches to solving unit problems may find themselves immersed in more pressing matters, which can lead 
to a rapid demise of innovative solutions. Not only are there challenges to sustaining EBP projects that have demonstrated their value to the unit or hospital, but the forward momentum for early-stage projects can be halted very quickly when champions are required to relocate or perhaps to leave the Army altogether. One sustainment strategy that has worked well in some organizations is to pair professionals who represent the military and civilian workforce as partners in EBP projects to ensure continuity and longevity for successful solutions.

In their paper describing transformational efforts directed at EBP institutionalization in two hospital settings, Stetler, Ritchie, Rycroft-Malone, Schultz, and Charns (2009) summarized their findings with these reflections: (a) organizations that experience a highly receptive context for EBP are more likely to achieve a higher level of institutionalization; (b) efforts to transform an organization desiring EBP institutionalization require the proactive, meaningful engagement of formal and informal leaders at all levels of the organization, including and especially staff nurses; (c) executive leaders who have the ability to proactively influence an organization's culture to support EBP and who can protect the related strategic vision from periodic pressures are more likely to institutionalize EBP over time; and (d) inconsistent operationalization of EBP-related infrastructures by formal leaders will have a negative impact on an organization's ability to institutionalize EBP. These insightful observations point to the power of the organization's formal leaders, who can choose to accept the challenge to enforce widespread EBP adoption, which means consistently seeking the best evidence in all decision making to achieve positive outcomes, or not. Early nursing models describing EBP institutionalization did not identify leadership as significant to the process; however, newer models focusing on innovations in health care highlight the prominent role played by executive leadership (Stetler et al., 2009). Given the hierarchy of any military organization, new initiatives requiring widespread support and sustainment are best disseminated to leaders at all levels, to include the chief executive officer, senior department leaders, and middle managers. Reinforcement also requires a broad-scale effort touting success stories of PIs and garnering support for future IPE training and a culture of inquiry going forward.

\section{Conclusion}

This article describes the implementation of an evidence-based educational program to enhance staff professional performance and reduce professional errors in high-risk settings. Clinical nurse specialists and nurse scientists in the organization led the successful launch of the CTEP interprofessional education platform in 2015. These clinical experts are highly qualified to lead interprofessional teams and EBP education efforts striving to identify, reduce, and/or prevent breaches in safety and quality. Leaders have come to rely on the nurse experts to embrace the imperative of robust performance improvement and thus design EBP projects that advance the culture of safety. The institution's leaders continue to support the HRO-guided approach to advancing EBP, with several courses already under contract for 2018. The advanced practice nurses maintain regular contact with the EBP project leaders from each course for progress reports and troubleshooting, if needed. Most importantly, encouraging and supporting dissemination of win-win scenarios by abstract submissions to local and regional conferences, and through publications, is the final but perhaps most rewarding step in the process. The opportunity to stand before one's peers and describe a practice change that not only saved money for the hospital but also enhanced quality and safety through evidence-based care epitomizes the health system's motto of patient-centered in all we do. A small but dedicated interprofessional team successfully challenged hospital leaders to choose the path of becoming a continuously learning health system built on a foundation of care customized by the patient's needs and values with evidence-based decision making that promotes health. 


\section{Acknowledgment}

The authors are indebted to Dr. Lynn Gallagher-Ford for her expertise in EBP education and her meticulous review of this manuscript that included editorial corrections, as well as suggestions for improvement, given the journal readership

\section{References}

Agency for Healthcare Research and Quality. (2008). TeamSTEPPS national implementation project 2008. Retrieved October 30, 2017, from http://teamstepps.ahrq.gov/

Barr, H., Koppel, I., Reeves, S., Hammick, M., \& Freeth, D. (Eds.). (2005). Effective interprofessional education: Argument, assumption \& evidence. Oxford, UK: Blackwell. https://doi.org/10.1002/9780470776445

Blewett, L. A., Johnson, K., McCarthy, T., Lackner, T., \& Brandt, B. (2010). Improving geriatric transitional care through inter-professional care teams. Journal of Evaluation in Clinical Practice, 16(1), 57-63. https://doi.org/10.1111/j.1365-2753.2008.01114.x

Brashers, V. L., Curry, C. E., Harper, D. C., McDaniel, S. H., Pawlson, G., \& Ball, J. W. (2001). Interprofessional health care education: Recommendations of the National Academies of Practice Expert Panel on Health Care in the 21st Century. Issues in Interdisciplinary Care, 3(1), 21-31.

Buntin, M. B., Damberg, C., Haviland, A., Kapur, K., Lurie, N., McDevitt, R., \& Marquis, M. S. (2006). Consumerdirected health care: Early evidence about effects on cost and quality. Health Affairs, 25(6), w516-w530. https://doi.org/10.1377/hlthaff.25.w516

Cartwright, J., Franklin, D., Forman, D., \& Freegard, H. (2015). Promoting collaborative dementia care via online interprofessional education. Australasian Journal on Ageing, 34(2), 88-94. https://doi.org/10.1111/ajag.12106

Chassin, M. R., \& Loeb, J. M. (2013). High reliability health care: Getting there from here. The Milbank Quarterly, 91(3), 459-490. https://doi.org/10.1111/1468-0009.12023

Chen, A. K., Dennehy, C., Fitzsimmons, A., Hyde, S., Lee, K., Rivera, J., . . Wamsley, M. (2017). Teaching interprofessional collaborative care skills using a blended learning approach. Journal of Interprofessional Education \& Practice, 8, 86-90. https://doi.org/10.1016/j.xjep.2017.07.002

Coleman, M. T., McLean, A., Williams, L., \& Hasan, K. (2017). Improvement in interprofessional student learning and patient outcomes. Journal of Interprofessional Education \& Practice, 8, 28-33. https://doi.org/10.1016/j.xjep.2017.05

Evidence-Based Practice Centers (EPC) Program Overview. (2018). Content last reviewed January 2018. Agency for Healthcare Research and Quality, Rockville, MD. Retrieved March 9, 2018, from http://www.ahrq.gov/ research/findings/evidence-based-reports/overview/index.html

Fisher, M., Weyant, D., Sterrett, S., Ambrose, H., \& Apfel, A. (2017). Perceptions of interprofessional collaborative practice and patient/family satisfaction. Journal of Interprofessional Education \& Practice, 8, 95-102. https://doi.org/10.1016/j.xjep.2017.07.004

Freeth, D., Hammick, M., Reeves, S., Koppel, I., \& Barr, H. (Eds.). (2005). Effective interprofessional education: Development, delivery \& evaluation. Oxford, UK: Blackwell. https://doi.org/10.1002/9780470776438

Gallagher-Ford, L. (2014). Implementing and sustaining EBP in real world healthcare settings.

Transformational evidence-based leadership: Redesigning traditional roles to promote and sustain a culture of EBP. Worldviews on Evidence-Based Nursing, 11(2), 140-142. https://doi.org/10.1111/wvn.12033

Horoho, P. D. (2013). A system for health: Essential element of national security. U.S. Army Medical Department Journal, October-December, 4. 
Institute of Medicine. (2001). Crossing the quality chasm: A new health system for the 21 st century.

Washington DC: National Academy of Sciences.

Institute of Medicine Committee on the Health Professions Education Summit. (2003). Executive summary.

In A. C. Greiner \& E. Knebel (Eds.), Health professions education: A bridge to quality (pp. 1-13)

Washington, DC: National Academy Press.

Institute of Medicine Roundtable on Evidence-Based Medicine. (2009). Leadership commitments to improve

value in healthcare. Finding common ground: Workshop summary. Washington, DC: National Academies

Press. Available from https:/www.ncbi.nlm.nih.gov/books/NBK52847/

Interprofessional Education Collaborative Core Competencies for Interprofessional Collaborative Practice. (2011).

Report of an expert panel. Washington, DC: Interprofessional Education Collaborative Expert Panel.

James, J. (2013). A new, evidence-based estimate of patient harms associate with hospital care. Journal of Patient

Safety, 9(3), 122-128. https://doi.org/10.1097/PTS.0b013e3182948a69

Joint Commission. (2008, July 9). Behaviors that undermine a culture of safety (Sentinel Event Alert No. 40).

Retrieved from http://www.jointcommission.org/assets/1/18/SEA_40.pdf

King, G., Shaw, L., Orchard, C., \& Miller, S. (2010). The Interprofessional Socialization and Valuing Scale: A tool for evaluating the shift toward collaborative care approaches in health care settings. Work, 35, 77-85.

Madigan Army Medical Center. (2018). External website: http://www.mamc.health.mil/

Accessed September 18, 2018.

Makic, M. B. F., \& Wald, H. (2017). Achieving improved patient outcomes through interprofessional teams. Journal of Interprofessional Education \& Practice, 8, 91-94. https://doi.org/10.1016/j.xjep.2017.07.003

McCann, E. (2014). Deaths by medical mistakes hit records. HealthcareITNews, July 18. Retrieved October 31, 2017, from http://www.healthcareitnews.com/news/deaths-by-medical-mistakes-hit-records

Melnyk, B. M. (2012). Achieving a high-reliability organization through implementation of the ARCC model for systemwide sustainability of evidence-based practice. Nursing Administration Quarterly, 36(2), 127-135. https://doi.org/10.1097/NAQ.0b013e318249fb6a

Melnyk, B. M., \& Fineout-Overholt, E. (2002). Putting research into practice. Reflections on Nursing Leadership, 28(2), 22-25.

Melnyk, B. M., \& Fineout-Overholt, E. (2011). Evidence-based practice in nursing \& healthcare (2nd ed.). Philadelphia, PA: Wolters Kluwer / Lippincott Williams and Wilkins Health.

Melnyk, B. M., \& Fineout-Overholt, E. (2015). Evidence-based practice in nursing healthcare: A guide to best practice (3rd ed.). Philadelphia, PA: Wolters Kluwer.

Melnyk, B. M., Fineout-Overholt, E., Gallagher-Ford, L., \& Kaplan, L. (2012). The state of evidence-based practice in US nurses. Journal of Nursing Administration, 42(9), 410-417. https://doi.org/10.1097/NNA.0b013e3182664e0a

McGinty, J., \& Anderson, G. (2008). Predictors of physician compliance with American Heart Association guidelines for acute myocardial infarction. Critical Care Nursing Quarterly, 31(2), 161-172. https://doi.org/10.1097/01.CNQ.0000314476.64377.12

Patelarou, A. E., Kyriakoulis, K. G., Stamou, A. A., Laliotis, A., Sifaki-Pistolla, D., Matalliotakis, M., . . Patelarou, E. (2017). Approaches to teach evidence-based practice among health professionals: an overview ofthe existing evidence. Advances in Medical Education and Practice, 8, 455-464. https://doi.org/10.2147/AMEP.S134475

Peterson, J. A., \& Brommelsiek, M. (2017). Interprofessional education to foster communication and resilience among health professional students. Journal of Interprofessional Education \& Practice, 7, 1-3. https://doi.org/10.1016/j.xjep.2017.01.001 
Serksnys, D., Nanchal, R., \& Fletcher, K. E. (2017). Opportunities for interprofessional input into nurse and physician hand-off communication. Journal of Critical Care, 38, 47-51. https://doi.org/10.1016/j.jcrc.2016.09.004

Shortell, S. M., Rundall, T. G., \& Hsu, J. (2007). Improving patient care by linking evidence-based medicine and evidence-based management. Journal of the American Medical Association, 298(6), 673-676. https://doi.org/10.1001/jama.298.6.673

Stetler, C. B., Ritchie, J. A., Rycroft-Malone, J., Schultz, A. A., \& Charns, M. P. (2009). Institutionalizing evidence-based practice: An organizational case study using a model of strategic change. Implementation Science, 4, 78. https://doi.org/10.1186/1748-5908-4-78

Titler, M. G., Kleiber, C., Steelman, V. J., Rakel, B. A., Budreau, G., Everett, L. Q., . . Goode, C. (2001). The Iowa model of evidence-based practice to promote quality care. Critical Care Nursing Clinics of Nort h America, 13(4), 497-509. https://doi.org/10.1016/S0899-5885(18)30017-0

Weick, K. E., \& Sutcliffe, K. M. (2007). Managing the unexpected (2nd ed.). San Francisco, CA: Jossey-Bass.

Williams, D. O. (2004). Treatment delayed is treatment denied. Circulation, 109(15), 1806-1808. https://doi.org/10.1161/01.CIR.0000126892.17646.83

World Health Organization. (1988). Learning together to work together for health. Report of a WHO Study Group on multiprofessional education for health personnel: The team approach (Technical Report Series No. 769). Geneva, Switzerland: Author.

World Health Organization. (2010). Framework for action on interprofessional education \& collaborative practice (WHO Reference No. HRH/HPN/10.3). Geneva, Switzerland: Author. Available at http://www.who.int/hrh/resources/framework_action/en/ 